Nonlocal Cauchy problems for fractional evolution equations involving Volterra-Fredholm type integral operators

JinRong Wang, Wei Wei, and Michal Fečkan 


\title{
NONLOCAL CAUCHY PROBLEMS FOR FRACTIONAL EVOLUTION EQUATIONS INVOLVING VOLTERRA-FREDHOLM TYPE INTEGRAL OPERATORS
}

\author{
JINRONG WANG, WEI WEI, AND MICHAL FEČKAN \\ Received 19 December, 2011
}

\begin{abstract}
In this paper, nonlocal Cauchy problems for fractional evolution equations involving Volterra-Fredholm type integral operators are investigated. Some new existence theorems of mild solutions are presented by using fractional calculus, Hölder inequality, Beta function and fixed point theorems.
\end{abstract}

2000 Mathematics Subject Classification: 26A33; 47J35

Keywords: fractional evolution equations, nonlocal Cauchy problems, mild solutions, analytic compact semigroup, fixed point theorems

\section{INTRODUCTION}

The fractional differential equations has recently been proved to be valuable tools in the modeling of many phenomena in various fields of engineering, physics, economy and science. We can find numerous applications in viscoelasticity, electrochemistry, control, porous media, electromagnetic, etc. [8,11,12, 14, 22, 23]. There has been a significant development in fractional differential equations. For more details on fractional calculus theory, one can see the monographs of Diethelm [9], Kilbas et al. [17], Lakshmikantham et al. [19], Michalski [24], Miller and Ross [25], Podlubny [29] and Tarasov [30]. In the last years, the theory of fractional differential equations attracted the attention of many authors (see for instance $[1-7,10,13,15,16$, 21,26, 27, 32, 34, 35] and references therein).

However, to our knowledge, nonlocal Cauchy problems for fractional evolution equations involving Volterra-Fredholm type integral operators has not been discussed extensively. Motivated by the above mentioned works (including our papers [6,

The first and second authors acknowledge the support by Key Projects of Science and Technology Research in the Chinese Ministry of Education (211169), Natural Science Foundation of Guizhou Province (2010, No.2142). The third author acknowledges the support by Grants VEGA-MS 1/0507/11, VEGA-SAV 2/0124/10 and APVV-0134-10. 
$20,21,26,31-33,35])$, the main purpose of this paper is to consider the following nonlocal Cauchy problems for fractional evolution equations involving VolterraFredholm type integral operators such as

$$
\left\{\begin{array}{c}
{ }^{c} D_{t}^{q} x(t)=-A x(t)+t^{n} f(t, x(t),(K x)(t),(H x)(t)), \\
\quad t \in J=[0, T], n \in Z^{+}, q \in(0,1), \\
x(0)=g(x)+x_{0},
\end{array}\right.
$$

where the fractional derivative ${ }^{c} D_{t}^{q}$ is understood here in the Caputo sense, $-A$ : $D(A) \rightarrow X$ is the infinitesimal generator of a compact analytic semigroup of uniformly bounded linear operators $\{S(t), t \geq 0\}$, the Volterra type integral operator $K$ and Fredholm type integral operator $H$ are defined by

$$
(K x)(t)=\int_{0}^{t} k(t, s, x(s)) d s, \quad(H x)(t)=\int_{0}^{T} h(t, s, x(s)) d s .
$$

The function $f: J \times X_{\alpha} \times X_{\alpha} \times X_{\alpha} \rightarrow X\left(\right.$ or $X_{\alpha}$, or $\left.X_{\mu}\right)$ is continuous where $X_{\alpha}=$ $D\left(A^{\alpha}\right), 0 \leq \mu \leq \alpha \leq 1$, is a Banach space with the norm $\|x\|_{\alpha}=\left\|A^{\alpha} x\right\|$ for $x \in X_{\alpha}$. $f, k, h$ and $g$ are specified latter. It is easy to see that term $t^{n}$ appears before the nonlinear term $f$. We remark that this term $t^{n}$ will help us to overcome the essential difficult caused by the singular term $(t-s)^{q-1}$ in the formula of the solutions due to the well known Beta function.

In the present paper, we discuss the existence and uniqueness of mild solutions for system (1.1). Our results cover the cases for the nonlinear term $f$ taking values in the spaces such as $X, X_{\alpha}, X_{\mu}$, where $0 \leq \mu \leq \alpha \leq 1$, the nonlocal term $g$ is linear completely continuous or satisfies the Lipschitz continuous condition. The main techniques used here are Hölder's inequality, Beta function via Banach contraction principle, Schauder's fixed point theorem for compact maps and Sadovskii's fixed point theorem for condensing maps.

The rest of this paper is organized as follows. In Section 2, we give some known preliminary results on the fraction powers of the generator of an analytic compact semigroup and introduce the mild solution of system (1.1). In Section 3, we study the existence of mild solutions for system (1.1) by using fractional calculus, Hölder inequality via Banach contraction principle, Schauder's fixed point theorem and Sadovskii's fixed point theorem. At last, an example is given to demonstrate the applicability of our result.

\section{PRELIMinaries}

In this section, we introduce some facts about the fractional powers of the generator of a compact analytic semigroup, the Riemann-Liouville fractional integral operator that are used throughout this paper.

We denote by $X$ a Banach space with norm $\|\cdot\|$ and $-A: D(A) \rightarrow X$ is the infinitesimal generator of a compact analytic semigroup of uniformly bounded linear operators $\{S(t), t \geq 0\}$. This means that there exists $M>1$ such that $\|S(t)\| \leq M$. 
We assume without loss of generality that $0 \in \rho(A)$. This allows us to define the fractional power $A^{\alpha}$ for $0 \leq \alpha \leq 1$, as a closed linear operator on its domain $D\left(A^{\alpha}\right)$ with inverse $A^{-\alpha}$ (see [28]).

In the sequel, we will also use $\|l\|_{L^{p}\left(J, R^{+}\right)}$to denote the $L^{p}\left(J, R^{+}\right)$norm of $l$ whenever $l \in L^{p}\left(J, R^{+}\right)$for some $p$ with $1 \leq p<\infty$. We will set $\alpha \in[0,1]$ and denote by $C_{\alpha}$, the Banach space $C\left(J, X_{\alpha}\right)$ endowed with supnorm given by $\|x\|_{\infty}=$ $\sup _{t \in J}\|x\|_{\alpha}$, for $x \in C_{\alpha}$.

Let us recall the following known definitions. For more details, see [17].

Definition 1. The fractional integral of order $\gamma$ with lower limit zero for a function $l$ is defined as

$$
I^{\gamma} l(t)=\frac{1}{\Gamma(\gamma)} \int_{0}^{t} \frac{l(s)}{(t-s)^{1-\gamma}} d s, t>0, \gamma>0,
$$

provided the right side is point-wise defined on $[0, \infty)$, where $\Gamma(\cdot)$ is the gamma function.

Definition 2. The Riemann-Liouville derivative of order $\gamma$ with lower limit zero for a function $l:[0, \infty) \rightarrow R$ can be written as

$$
{ }^{L} D_{t}^{\gamma} l(t)=\frac{1}{\Gamma(n-\gamma)} \frac{d^{n}}{d t^{n}} \int_{0}^{t} \frac{l(s)}{(t-s)^{\gamma+1-n}} d s, t>0, n-1<\gamma<n .
$$

Definition 3. The Caputo derivative of order $\gamma$ for a function $l:[0, \infty) \rightarrow R$ can be written as

$$
{ }^{c} D_{t}^{\gamma} l(t)={ }^{L} D^{\gamma}\left(l(t)-\sum_{k=0}^{n-1} \frac{t^{k}}{k !} l^{(k)}(0)\right), t>0, n-1<\gamma<n .
$$

Remark 1. (i) If $l \in C^{1}[0, \infty)$, then

$$
{ }^{c} D_{t}^{\gamma} l(t)=\frac{1}{\Gamma(1-\gamma)} \int_{0}^{t} \frac{l^{\prime}(s)}{(t-s)^{\gamma}} d s=I^{1-\gamma} l^{\prime}(t), t>0,0<\gamma<1 .
$$

(ii) The Caputo derivative of a constant is equal to zero.

(iii) If $l$ is an abstract function with values in $X$, then integrals which appear in Definitions 1 and 2 are taken in Bochner's sense.

Motivated by Definition 3.1 of [35], we adopt the following concept of mild solution for our problem.

Definition 4. By the mild solution of system (1.1), we mean that the function $x: J \rightarrow X_{\alpha}$ which satisfies

$$
x(t)=\mathscr{T}(t)\left[x_{0}+g(x)\right]+\int_{0}^{t}(t-s)^{q-1} s^{n} \mathscr{S}(t-s) f(s, x(s),(K x)(s),(H x)(s)) d s
$$


for any $t \in J$, where

$$
\mathscr{T}(t)=\int_{0}^{\infty} \xi_{q}(\theta) S\left(t^{q} \theta\right) d \theta, \quad \mathscr{S}(t)=q \int_{0}^{\infty} \theta \xi_{q}(\theta) S\left(t^{q} \theta\right) d \theta
$$

and

where

$$
\xi_{q}(\theta)=\frac{1}{q} \theta^{-1-\frac{1}{q}} \varpi_{q}\left(\theta^{-\frac{1}{q}}\right) \geq 0
$$

$$
\varpi_{q}(\theta)=\frac{1}{\pi} \sum_{n=1}^{\infty}(-1)^{n-1} \theta^{-q n-1} \frac{\Gamma(n q+1)}{n !} \sin (n \pi q), \quad \theta \in(0, \infty) .
$$

$\xi_{q}$ is a probability density function defined on $(0, \infty)$, that is

$$
\xi_{q}(\theta) \geq 0, \quad \theta \in(0, \infty) \quad \text { and } \quad \int_{0}^{\infty} \xi_{q}(\theta) d \theta=1 .
$$

Remark 2. It is not difficult to verify that for $v \in[0,1]$,

$$
\int_{0}^{\infty} \theta^{v} \xi_{q}(\theta) d \theta=\int_{0}^{\infty} \theta^{-q v} \varpi_{q}(\theta) d \theta=\frac{\Gamma(1+v)}{\Gamma(1+q v)} .
$$

The following results are very useful and will be used throughout this paper.

Lemma 1 (Lemma 2.9, [32]). The operators $\mathscr{T}$ and $\mathscr{S}$ have the following properties:

(1) For fixed $t \geq 0, \mathscr{T}(t)$ and $\mathscr{S}(t)$ are linear and bounded operators, that is, for any $x \in X$,

$$
\|\mathscr{T}(t) x\| \leq M\|x\|, \quad\|\mathscr{S}(t) x\| \leq \frac{M}{\Gamma(q)}\|x\| .
$$

(2) $\{\mathscr{T}(t), t \geq 0\}$ and $\{\mathscr{S}(t), t \geq 0\}$ are strongly continuous.

(3) For every $t>0, \mathscr{T}(t)$ and $\mathscr{S}(t)$ are also compact operators.

(4) For any $x \in X, \beta \in[0,1]$ and $\alpha \in[0,1]$, we have

$$
\begin{gathered}
A \mathscr{S}(t) x=A^{1-\beta} \mathscr{S}(t) A^{\beta} x, \quad t \in J, \\
\left\|A^{\alpha} \mathscr{S}(t)\right\| \leq \frac{M_{\alpha} q \Gamma(2-\alpha)}{\Gamma(1+q(1-\alpha))} t^{-\alpha q}, \quad 0<t \leq T .
\end{gathered}
$$

(5) For fixed $t \geq 0$ and any $x \in X_{\alpha}$, we have

$$
\|\mathscr{T}(t) x\|_{\alpha} \leq M\|x\|_{\alpha}, \quad\|\mathscr{S}(t) x\|_{\alpha} \leq \frac{M}{\Gamma(q)}\|x\|_{\alpha} .
$$

(6) For a positive number $\mu$ with $0 \leq \mu \leq \alpha \leq 1$, fixed $t \geq 0$ and any $x \in X_{\mu}$, we have

$\|\mathscr{T}(t) x\|_{\alpha} \leq\left\|A^{\alpha-\mu}\right\| M\|x\|_{\mu}, \quad\|\mathscr{S}(t) x\|_{\alpha} \leq\left\|A^{\alpha-\mu}\right\| \frac{M}{\Gamma(q)}\|x\|_{\mu}$. 
(7) $\mathscr{T}_{\alpha}(t)$ and $\mathscr{S}_{\alpha}(t), t>0$ is uniformly continuous, that is for each fixed $t>0$, and $\epsilon>0$, there exists $h>0$ such that

$$
\begin{aligned}
& \left\|\mathscr{T}_{\alpha}(t+\epsilon)-\mathscr{T}_{\alpha}(t)\right\|_{\alpha}<\varepsilon, \quad \text { for } \quad t+\epsilon \geq 0 \text { and } \quad|\epsilon|<h \text {, } \\
& \left\|\mathscr{S}_{\alpha}(t+\epsilon)-\mathscr{S}_{\alpha}(t)\right\|_{\alpha}<\varepsilon, \quad \text { for } t+\epsilon \geq 0 \text { and }|\epsilon|<h \text {. }
\end{aligned}
$$

where

$$
\mathscr{T}_{\alpha}(t)=\int_{0}^{\infty} \xi_{q}(\theta) S_{\alpha}\left(t^{q} \theta\right) d \theta, \quad \mathscr{S}_{\alpha}(t)=q \int_{0}^{\infty} \theta \xi_{q}(\theta) S_{\alpha}\left(t^{q} \theta\right) d \theta .
$$

\section{EXISTENCE OF MILD SOLUTIONS}

In this section, we give theorems for the existence and uniqueness of the mild solutions of system (1.1).

We first make the following assumptions.

[Hf1]: $f: J \times X_{\alpha} \times X_{\alpha} \times X_{\alpha} \rightarrow X$ is continuous and there exist $m_{1}, m_{2}, m_{3}>0$ such that

$\left\|f\left(t, x_{1}, x_{2}, x_{3}\right)-f\left(t, y_{1}, y_{2}, y_{3}\right)\right\| \leq m_{1}\left\|x_{1}-y_{1}\right\|_{\alpha}+m_{2}\left\|x_{2}-y_{2}\right\|_{\alpha}+m_{3}\left\|x_{3}-y_{3}\right\|_{\alpha}$ for all $x_{i}, y_{i} \in X_{\alpha}, i=1,2,3$ and $t \in J$.

[Hk1]: Let $D_{k}=\left\{(t, s) \in R^{2} ; 0 \leq s \leq t \leq T\right\}$. The function $k: D_{k} \times X_{\alpha} \rightarrow X_{\alpha}$ is continuous and there exists a $m_{k}(t, s) \in C\left(D_{k}, R^{+}\right)$such that

$$
\|k(t, s, x)-k(t, s, y)\|_{\alpha} \leq m_{k}(t, s)\|x-y\|_{\alpha}
$$

for each $(t, s) \in D_{k}$ and $x, y \in X_{\alpha}$. We set

$$
K^{*}=\max _{t \in J} \int_{0}^{t} m_{k}(t, s) d s .
$$

[Hh1]: Let $D_{h}=\left\{(t, s) \in R^{2} ; 0 \leq s, t \leq T\right\}$. The function $h: D_{h} \times X_{\alpha} \rightarrow X_{\alpha}$ is continuous and there exists a $m_{h}(t, s) \in C\left(D_{h}, R^{+}\right)$such that

$$
\|h(t, s, x)-h(t, s, y)\|_{\alpha} \leq m_{h}(t, s)\|x-y\|_{\alpha}
$$

for each $(t, s) \in D_{h}$ and $x, y \in X_{\alpha}$. We set

$$
H^{*}=\max _{t \in J} \int_{0}^{T} m_{h}(t, s) d s .
$$

[Hg1]: $g: C_{\alpha} \rightarrow X_{\alpha}$ and there exists a constant $l_{g}>0$ such that

$$
\|g(x)-g(y)\|_{\alpha} \leq l_{g}\|x-y\|_{\infty}, \text { for arbitrary } x, y \in C_{\alpha} .
$$

[H $\Omega]$ : A constant $\Omega_{n, \alpha, q, T}$ defined by

$$
\Omega_{n, \alpha, q, T}=M l_{g}+\frac{M_{\alpha} q \Gamma(2-\alpha) B(q, n+1)}{\Gamma(1+q(1-\alpha))} T^{n+(1-\alpha) q}\left(m_{1}+K^{*} m_{2}+H^{*} m_{3}\right)
$$

satisfies $\Omega_{n, \alpha, q, T}<1$, where $B(\cdot, \cdot)$ denotes Beta function. 
Now we are ready to give our first result which is based on the Banach contraction mapping principle.

Theorem 1. Assume that [Hfl], [Hk1], [Hhl], [Hgl] and [HS] are satisfied. If $x_{0} \in X_{\alpha}$ then system (1.1) has a unique mild solution $x \in C_{\alpha}$.

Proof. Define the function $\Gamma: C_{\alpha} \rightarrow C_{\alpha}$ by

$$
\begin{aligned}
(\Gamma x)(t)= & \mathscr{T}(t)\left[x_{0}+g(x)\right] \\
& +\int_{0}^{t}(t-s)^{q-1} s^{n} \mathscr{S}(t-s) f(s, x(s),(K x)(s),(H x)(s)) d s, t \in J .
\end{aligned}
$$

Note that $\Gamma$ is well defined on $C_{\alpha}$. Now, take $t \in J$ and $x, y \in C_{\alpha}$. Then we have

$$
\begin{gathered}
\|(\Gamma x)(t)-(\Gamma y)(t)\|_{\alpha} \leq\|\mathscr{T}(t)(g(x)-g(y))\|_{\alpha} \\
+\int_{0}^{t}(t-s)^{q-1} s^{n} \| \mathscr{S}(t-s)[f(s, x(s),(K x)(t),(H x)(t)) \\
\left.\leq M\|g(x)-g(y)\|_{\alpha} \quad-f(s, y(s),(K y)(s),(H y)(s))\right] \|_{\alpha} d s \\
+\int_{0}^{t}(t-s)^{q-1} s^{n}\left\|A^{\alpha} \mathscr{S}(t-s)\right\| \| f(s, x(s),(K x)(s),(H x)(s)) \\
-f(s, y(s),(K y)(s),(H y)(s)) \| d s,
\end{gathered}
$$

which according to [Hf1], [Hk1], [Hh1], [Hg1], (4)-(5) of Lemma 1 and Hölder inequality,gives

$$
\begin{aligned}
\| & (\Gamma x)(t)-(\Gamma y)(t)\left\|_{\alpha} \leq M l_{g}\right\| x-y \|_{\infty} \\
& +M_{\alpha} q t^{-\alpha q} \frac{\Gamma(2-\alpha)}{\Gamma(1+q(1-\alpha))} m_{1} \int_{0}^{t}(t-s)^{q-1} s^{n}\|x(s)-y(s)\|_{\alpha} d s \\
& +M_{\alpha} q t^{-\alpha q} \frac{\Gamma(2-\alpha)}{\Gamma(1+q(1-\alpha))} m_{2} \int_{0}^{t}(t-s)^{q-1} s^{n}\|(K x)(s)-(K y)(s)\|_{\alpha} d s \\
& +M_{\alpha} q t^{-\alpha q} \frac{\Gamma(2-\alpha)}{\Gamma(1+q(1-\alpha))} m_{3} \int_{0}^{t}(t-s)^{q-1} s^{n}\|(H x)(s)-(H y)(s)\|_{\alpha} d s \\
\leq & M l_{g}\|x-y\|_{\infty}+M_{\alpha} q t^{-\alpha q} \frac{\Gamma(2-\alpha)}{\Gamma(1+q(1-\alpha))}\left(m_{1}+K^{*} m_{2}+H^{*} m_{3}\right) \\
& \times\|x-y\|_{\infty} \int_{0}^{t}(t-s)^{q-1} s^{n} d s \\
\leq & \left\{M l_{g}+\frac{M_{\alpha} q \Gamma(2-\alpha) B(q, n+1)}{\Gamma(1+q(1-\alpha))} t^{n+(1-\alpha) q}\left(m_{1}+K^{*} m_{2}+H^{*} m_{3}\right)\right\} \\
& \times\|x-y\|_{\infty},
\end{aligned}
$$


due to

$$
\int_{0}^{t}(t-s)^{q-1} s^{n} d s=B(q, n+1) t^{n+q}
$$

$\|(K x)(s)-(K y)(s)\|_{\alpha} \leq K^{*}\|x-y\|_{\infty}$ and $\|(H x)(s)-(H y)(s)\|_{\alpha} \leq H^{*}\|x-y\|_{\infty}$.

Therefore, we can deduce that

$$
\begin{aligned}
& \|\Gamma x-\Gamma y\|_{\infty} \\
& \leq\left\{M l_{g}+\frac{M_{\alpha} q \Gamma(2-\alpha) B(q, n+1)}{\Gamma(1+q(1-\alpha))} t^{n+(1-\alpha) q}\left(m_{1}+K^{*} m_{2}+H^{*} m_{3}\right)\right\} \\
& \quad \times\|x-y\|_{\infty} \leq \Omega_{n, \alpha, q, T}\|x-y\|_{\infty} .
\end{aligned}
$$

Hence, $[\mathrm{H} \Omega]$ allows us to conclude in view of the contraction mapping principle, that $\Gamma$ has a unique fixed point $x \in C_{\alpha}$, and

$x(t)=\mathscr{T}(t)\left[x_{0}+g(x)\right]+\int_{0}^{t}(t-s)^{q-1} s^{n} \mathscr{S}(t-s) f(s, x(s),(K x)(s),(H x)(s)) d s$

which is the unique mild solution of system (1.1).

Our second result uses Schauder's fixed point theorem.

We assume the following conditions.

[Hf2]: $f: J \times X_{\alpha} \times X_{\alpha} \times X_{\alpha} \rightarrow X_{\alpha}$ is continuous and there exists a positive function $\rho \in L^{p}\left(J, R^{+}\right)$for some $p \in\left(\frac{1}{q}, \infty\right)$ such that

$$
\|f(t, x, y, z)\|_{\alpha} \leq \rho(t)
$$

for all $x, y, z \in X_{\alpha}$ and $t \in J$.

[Hk2]: The function $k: D_{k} \times X_{\alpha} \rightarrow X_{\alpha}$ is continuous and there exists $L_{1}>0$ such that

$$
\|k(t, s, x)-k(t, s, y)\|_{\alpha} \leq L_{1}\|x-y\|_{\alpha}
$$

for each $(t, s) \in D_{k}$ and $x, y \in X_{\alpha}$.

[Hh2]: The function $h: D_{h} \times X_{\alpha} \rightarrow X_{\alpha}$ is continuous and there exists $L_{2}>0$ such that

$$
\|h(t, s, x)-h(t, s, y)\|_{\alpha} \leq L_{2}\|x-y\|_{\alpha}
$$

for each $(t, s) \in D_{h}$ and $x, y \in X_{\alpha}$. that

[Hg2]: $g: C_{\alpha} \rightarrow X_{\alpha}$ is compact continuous and there exist $\beta_{1} \geq 0, \beta_{2} \geq 0$ such

$$
\|g(x)\|_{\alpha} \leq \beta_{1}\|x\|_{\infty}+\beta_{2} .
$$

Now we are ready to state and prove the following existence result.

Theorem 2. Assume that the conditions [Hf2], [Hk2], [Hh2], [Hg2] are satisfied. If $x_{0} \in X_{\alpha}$ then system (1.1) has at least one mild solution on J provided that

$$
M \beta_{1}<1 \text {. }
$$


Proof. Define the function $F: C_{\alpha} \rightarrow C_{\alpha}$ by

$$
\begin{aligned}
(F x)(t)= & \mathscr{T}(t)\left[x_{0}+g(x)\right] \\
& +\int_{0}^{t}(t-s)^{q-1} s^{n} \mathscr{S}(t-s) f(s, x(s),(K x)(s),(H x)(s)) d s,
\end{aligned}
$$

and for $n \in Z^{+}$, we choose $r$ such that

$$
\begin{aligned}
r \geq & \frac{1}{1-M \beta_{1}}\left[M\left(\left\|x_{0}\right\|_{\alpha}+\beta_{2}\right)\right. \\
& \left.+\frac{M}{\Gamma(q)} B\left(\frac{p q-1}{p-1}, \frac{(n+1) p-1}{p-1}\right)^{\frac{p-1}{p}} T^{\frac{p q+n p-1}{p}}\|\rho\|_{L^{p}\left(J, R^{+}\right)}\right] .
\end{aligned}
$$

Let $B_{r}=\left\{x \in C_{\alpha} \mid\|x\|_{\infty} \leq r\right\}$. Then we proceed in three steps.

Step 1. We show that $F B_{r} \subset B_{r}$. Let $x \in B_{r}$. Then for $t \in J$, using (5) of Lemma 1 and Hölder inequality, we have

$$
\begin{aligned}
\|(F x)(t)\|_{\alpha} \leq & \left\|\mathscr{T}(t)\left(x_{0}+g(x)\right)\right\|_{\alpha} \\
& +\int_{0}^{t}(t-s)^{q-1} s^{n}\|\mathscr{S}(t-s) f(s, x(s),(K x)(s),(H x)(s))\|_{\alpha} d s \\
\leq & M\left(\left\|x_{0}\right\|_{\alpha}+\|g(x)\|_{\alpha}\right) \\
& +\frac{M}{\Gamma(q)} \int_{0}^{t}(t-s)^{q-1} s^{n}\|f(s, x(s),(K x)(s),(H x)(s))\|_{\alpha} d s,
\end{aligned}
$$

which according to [Hf2], [Hg2] and $p q>1\left(\Leftrightarrow \frac{(q-1) p}{p-1}>-1\right)$, gives

$$
\begin{aligned}
\| & (F x)(t) \|_{\alpha} \\
\leq & M\left(\left\|x_{0}\right\|_{\alpha}+\beta_{1}\|x\|_{\infty}+\beta_{2}\right)+\frac{M}{\Gamma(q)}\left(\int_{0}^{t}(t-s)^{q-1} s^{n} \rho(s) d s\right) \\
\leq & M\left(\left\|x_{0}\right\|_{\alpha}+\beta_{1}\|x\|_{\infty}+\beta_{2}\right)+\frac{M}{\Gamma(q)}\left(\int_{0}^{t}(t-s)^{\frac{(q-1) p}{p-1}} s^{\frac{n p}{p-1}} d s\right)^{\frac{p-1}{p}} \\
& \times\left(\int_{0}^{t} \rho(s)^{p} d s\right)^{\frac{1}{p}} \\
\leq & M\left(\left\|x_{0}\right\|_{\alpha}+\beta_{1}\|x\|_{\infty}+\beta_{2}\right)+\frac{M}{\Gamma(q)} B\left(\frac{p q-1}{p-1}, \frac{(n+1) p-1}{p-1}\right)^{\frac{p-1}{p}} \\
& \times t^{\frac{p q+n p-1}{p}}\|\rho\|_{L^{p}\left(J, R^{+}\right)} \leq r, \text { for } t \in J .
\end{aligned}
$$

Hence, we deduce $\|F x\|_{\infty} \leq r$.

Step 2. We prove that $F$ is continuous. Let $\left\{x_{m}\right\}$ be a sequence of $B_{r}$ such that $x_{m} \rightarrow x$ in $B_{r}$. It comes from the continuity of $k, h$ and assumptions [Hk2], [Hh2] 
that

$$
\begin{aligned}
\int_{0}^{s} k\left(s, \tau, x_{m}(\tau)\right) d \tau & \rightarrow \int_{0}^{s} k(s, \tau, x(\tau)) d \tau \\
\int_{0}^{T} h\left(s, \tau, x_{m}(\tau)\right) d \tau & \rightarrow \int_{0}^{T} h(s, \tau, x(\tau)) d \tau
\end{aligned}
$$

uniformly in $s \in J$ on $C_{\alpha}$. Then,

$$
f\left(s, x_{m}(s),\left(K x_{m}\right)(s),\left(H x_{m}\right)(s)\right) \rightarrow f(s, x(s),(K x)(s),(H x)(s))
$$

as $m \rightarrow \infty$, because the function $f$ is continuous on $J \times X_{\alpha} \times X_{\alpha}$.

Further, one has

$$
g\left(x_{m}\right) \rightarrow g(x) \text { as } m \rightarrow \infty
$$

because $g$ is continuous on $C_{\alpha}$.

Now for $t \in J$, according to [Hf2], [Hg2], (5) of Lemma 1 and Hölder inequality, we have

$$
\begin{aligned}
& \left\|\left(F x_{m}\right)(t)-(F x)(t)\right\|_{\alpha} \leq\left\|\mathscr{T}(t)\left(g\left(x_{m}\right)-g(x)\right)\right\|_{\alpha} \\
& +\int_{0}^{t}(t-s)^{q-1} s^{n} \| \mathscr{S}(t-s)\left[f\left(s, x_{m}(s),\left(K x_{m}\right)(s),\left(H x_{m}\right)(s)\right)\right. \\
& -f(s, x(s),(K x)(s),(H x)(s))] \|_{\alpha} d s \\
& \leq M\left\|g\left(x_{m}\right)-g(x)\right\|_{\alpha} \\
& +\frac{M}{\Gamma(q)} \int_{0}^{t}(t-s)^{q-1} s^{n} \| f\left(s, x_{m}(s),\left(K x_{m}\right)(s),\left(H x_{m}\right)(s)\right) \\
& -f(s, x(s),(K x)(s),(H x)(s)) \|_{\alpha} d s \\
& \leq M\left\|g\left(x_{m}\right)-g(x)\right\|_{\alpha}+\frac{M}{\Gamma(q)}\left(\int_{0}^{t}(t-s)^{\frac{(q-1) p}{p-1}} s^{\frac{n p}{p-1}} d s\right)^{\frac{p-1}{p}} \\
& \times\left(\int_{0}^{t} \| f\left(s, x_{m}(s),\left(K x_{m}\right)(s),\left(H x_{m}\right)(s)\right)\right. \\
& \left.-f(s, x(s),(K x)(s),(H x)(s)) \|^{p} d s\right)^{\frac{1}{p}} \\
& \leq M\left\|g\left(x_{m}\right)-g(x)\right\|_{\alpha}+\frac{M}{\Gamma(q)} B\left(\frac{p q-1}{p-1}, \frac{(n+1) p-1}{p-1}\right)^{\frac{p-1}{p}} t^{\frac{p q+n p-1}{p}} \\
& \times\left(\int_{0}^{t} \| f\left(s, x_{m}(s),\left(K x_{m}\right)(s),\left(H x_{m}\right)(s)\right)\right.
\end{aligned}
$$




$$
\begin{gathered}
\left.-f(s, x(s),(K x)(s),(H x)(s)) \|^{p} d s\right)^{\frac{1}{p}} \\
\leq M\left\|g\left(x_{m}\right)-g(x)\right\|_{\alpha}+\frac{M}{\Gamma(q)} B\left(\frac{p q-1}{p-1}, \frac{(n+1) p-1}{p-1}\right)^{\frac{p-1}{p}} T^{\frac{p q+n p-1}{p}} \\
\times\left(\int_{0}^{T} \| f\left(s, x_{m}(s),\left(K x_{m}\right)(s),\left(H x_{m}\right)(s)\right)\right. \\
\left.-f(s, x(s),(K x)(s),(H x)(s)) \|^{p} d s\right)^{\frac{1}{p}} .
\end{gathered}
$$

Therefore, using (3.2), (3.3), [Hf2] and the Lebesgue Dominated Convergence Theorem, it can easily been shown that

$$
\lim _{m \rightarrow \infty}\left\|F x_{m}-F x\right\|_{\infty}=0, \text { as } m \rightarrow \infty .
$$

That is, $F$ is continuous.

Step 3. We show that $F$ is compact. To this end, we use the famous AscoliArzela's theorem. We first prove that $\left\{(F x)(t) \mid x \in B_{r}\right\}$ is relatively compact in $X_{\alpha}$, for all $t \in J$. Obviously, $\left\{(F x)(0) \mid x \in B_{r}\right\}$ is compact. Let $t \in(0, T]$. For each $h \in(0, t)$, arbitrary $\delta>0$ and $x \in B_{r}$, we defined the operator $F_{h}$ by

$$
\begin{gathered}
\left(F_{h, \delta} x\right)(t)=\mathscr{T}(t)\left[x_{0}+g(x)\right]+S\left(h^{q} \delta\right) \int_{0}^{t-h}(t-s)^{q-1} s^{n} \\
\times\left(q \int_{\delta}^{\infty} \theta \xi_{q}(\theta) S\left((t-s)^{q} \theta-h^{q} \delta\right) d \theta\right) f(s, x(s),(K x)(s),(H x)(s)) d s \\
=\mathscr{T}(t)\left[x_{0}+g(x)\right] \\
+q \int_{0}^{t-h} \int_{\delta}^{\infty} \theta(t-s)^{q-1} s^{n} \xi_{q}(\theta) S\left((t-s)^{q} \theta\right) \\
\times f(s, x(s),(K x)(s),(H x)(s)) d \theta d s .
\end{gathered}
$$

From above expression, we can see that the sets $\left\{\left(F_{h, \delta} x\right)(t) \mid x \in B_{r}\right\}$ are also relatively compact in $X_{\alpha}$ since the operator $S_{\alpha}\left(h^{q} \delta\right), h^{q} \delta>0$ are compact in $X_{\alpha}$. Moreover, using [Hf2] and Hölder inequality, we have

$$
\begin{aligned}
& \left\|(F x)(t)-\left(F_{h, \delta} x\right)(t)\right\|_{\alpha} \\
& \begin{array}{l}
\leq q \| \int_{0}^{t} \int_{0}^{\delta} \theta(t-s)^{q-1} s^{n} \xi_{q}(\theta) S\left((t-s)^{q} \theta\right) \\
\quad \times f(s, x(s),(K x)(s),(H x)(s)) d \theta d s \|_{\alpha}
\end{array}
\end{aligned}
$$




$$
\begin{aligned}
& +q \| \int_{0}^{t} \int_{\delta}^{\infty} \theta(t-s)^{q-1} s^{n} \xi_{q}(\theta) S\left((t-s)^{q} \theta\right) \\
& \times f(s, x(s),(K x)(s),(H x)(s)) d \theta d s \\
& -\int_{0}^{t-h} \int_{\delta}^{\infty} \theta(t-s)^{q-1} s^{n} \xi_{q}(\theta) S\left((t-s)^{q} \theta\right) \\
& \times f(s, x(s),(K x)(s),(H x)(s)) d \theta d s \|_{\alpha} \\
& \leq q \int_{0}^{t} \int_{0}^{\delta} \theta(t-s)^{q-1} s^{n} \xi_{q}(\theta) \| S\left((t-s)^{q} \theta\right) \\
& +q \int_{t-h}^{t} \quad \times f(s, x(s),(K x)(s),(H x)(s)) \|_{\alpha} d \theta d s \\
& \leq q M \int_{0}^{t} \int_{0}^{\delta} \theta(t-s)^{q-1} s^{n} \xi_{q}(\theta) \rho(s) d \theta d s \\
& +q M \int_{t-h}^{t} \int_{\delta}^{\infty} \theta(t-s)^{q-1} s^{n} \xi_{q}(\theta) \rho(s) d \theta d s \\
& \leq q M\left(\int_{0}^{t}(t-s)^{q-1} s^{n} \rho(s) d s\right) \int_{0}^{\delta} \theta \xi_{q}(\theta) d \theta \\
& +q M\left(\int_{t-h}^{t}(t-s)^{q-1} s^{n} \rho(s) d s\right) \int_{0}^{\infty} \theta \xi_{q}(\theta) d \theta .
\end{aligned}
$$

It comes from

$$
\int_{0}^{\infty} \theta \xi_{q}(\theta) d \theta=\frac{1}{\Gamma(1+q)}
$$

and

$$
\begin{gathered}
\int_{0}^{t}(t-s)^{q-1} s^{n} \rho(s) d s \leq\left(\int_{0}^{t}(t-s)^{\frac{(q-1) p}{p-1}} s^{\frac{n p}{p-1}} d s\right)^{\frac{p-1}{p}}\left(\int_{0}^{t} \rho(s)^{p} d s\right)^{\frac{1}{p}} \\
\leq B\left(\frac{p q-1}{p-1}, \frac{(n+1) p-1}{p-1}\right)^{\frac{p-1}{p}} t^{\frac{p q+n p-1}{p}}\|\rho\|_{L^{p}\left(J, R^{+}\right)},
\end{gathered}
$$


and for a fixed $\chi \in\left(1, \frac{p-1}{(1-q) p}\right)$,

$$
\begin{gathered}
\int_{t-h}^{t}(t-s)^{q-1} s^{n} \rho(s) d s \leq\left(\int_{t-h}^{t}(t-s)^{\frac{(q-1) p}{p-1}} s^{\frac{n p}{p-1}} d s\right)^{\frac{p-1}{p}}\left(\int_{t-h}^{t} \rho(s)^{p} d s\right)^{\frac{1}{p}} \\
\quad \leq\left(\int_{t-h}^{t} d s\right)^{\frac{\chi(p-1)}{(\chi-1) p}}\left(\int_{t-h}^{t}(t-s)^{\frac{\chi(q-1) p}{p-1}} s^{\frac{\chi n p}{p-1}} d s\right)^{\frac{p-1}{\chi p}}\|\rho\|_{L^{p}\left(J, R^{+}\right)} \\
\leq h^{\frac{\chi(p-1)}{(\chi-1) p}} B\left(\frac{\chi(q-1) p}{p-1}+1, \frac{\chi n p}{p-1}+1\right)^{\frac{p-1}{\chi p}} t^{\frac{\chi p((q-1) p+n)}{p-1}+1}\|\rho\|_{L^{p}\left(J, R^{+}\right)},
\end{gathered}
$$

that

$$
\begin{gathered}
\left\|(F x)(t)-\left(F_{h, \delta} x\right)(t)\right\|_{\alpha} \\
\leq q M B\left(\frac{p q-1}{p-1}, \frac{(n+1) p-1}{p-1}\right)^{\frac{p-1}{p}} t^{\frac{p q+n p-1}{p}}\|\rho\|_{L^{p}\left(J, R^{+}\right)} \int_{0}^{\delta} \theta \xi_{q}(\theta) d \theta \\
+\frac{M}{\Gamma(q)} B\left(\frac{\chi(q-1) p}{p-1}+1, \frac{\chi n p}{p-1}+1\right)^{\frac{p-1}{\chi p}} t^{\frac{\chi p((q-1) p+n)}{p-1}+1} \\
\times\|\rho\|_{L^{p}\left(J, R^{+}\right)} h^{\frac{\chi(p-1)}{(\chi-1) p}} \rightarrow 0
\end{gathered}
$$

as $\delta \rightarrow 0^{+}$and $h \rightarrow 0^{+}$. Therefore, $\left\{(F x)(t) \mid x \in B_{r}\right\}$ is relatively compact in $X_{\alpha}$ for all $t \in(0, T]$ and since it is compact at $t=0$ we have the relatively compactness in $X_{\alpha}$ for all $t \in J$.

Next, let us prove that $F\left(B_{r}\right)$ is equicontinuous. For $0 \leq t_{2}<t_{1} \leq T$, we have

$$
\begin{gathered}
\left\|(F x)\left(t_{1}\right)-(F x)\left(t_{2}\right)\right\|_{\alpha} \leq\left\|\left(\mathscr{T}\left(t_{1}\right)-\mathscr{T}\left(t_{2}\right)\right)\left[x_{0}-g(x)\right]\right\|_{\alpha} \\
+\| \int_{0}^{t_{2}}\left(t_{1}-s\right)^{q-1} s^{n}\left[\mathscr{S}\left(t_{1}-s\right)-\mathscr{S}\left(t_{2}-s\right)\right] \\
\times f(s, x(s),(K x)(s),(H x)(s)) d s \|_{\alpha} \\
\left.+\| \int_{0}^{t_{2}}\left[\left(t_{1}-s\right)^{q-1}-t_{2}-s\right)^{q-1}\right] s^{n} \mathscr{S}\left(t_{2}-s\right) \\
\times f(s, x(s),(K x)(s),(H x)(s)) d s \|_{\alpha} \\
+\left\|\int_{t_{2}}^{t_{1}}\left(t_{1}-s\right)^{q-1} s^{n} \mathscr{S}\left(t_{1}-s\right) f(s, x(s),(K x)(s),(H x)(s)) d s\right\|_{\alpha}
\end{gathered}
$$


Denote

$$
\begin{aligned}
& I_{1}=\left\|\left(\mathscr{T}\left(t_{1}\right)-\mathscr{T}\left(t_{2}\right)\right)\left[x_{0}-g(x)\right]\right\|_{\alpha}, \\
& \begin{aligned}
I_{2}=\| \int_{0}^{t_{2}}\left(t_{1}-s\right)^{q-1} s^{n}\left[\mathscr{S}\left(t_{1}-s\right)-\mathscr{S}\left(t_{2}-s\right)\right] \\
\quad \times f(s, x(s),(K x)(s),(H x)(s)) d s \|_{\alpha}, \\
I_{3}=\| \int_{0}^{t_{2}}\left[\left(t_{1}-s\right)^{q-1}-\left(t_{2}-s\right)^{q-1}\right] s^{n} \mathscr{S}\left(t_{2}-s\right) \\
\quad \times f(s, x(s),(K x)(s),(H x)(s)) d s \|_{\alpha}, \\
I_{4}=\left\|\int_{t_{2}}^{t_{1}}\left(t_{1}-s\right)^{q-1} s^{n} \mathscr{S}\left(t_{1}-s\right) f(s, x(s),(K x)(s),(H x)(s)) d s\right\|_{\alpha} .
\end{aligned}
\end{aligned}
$$

Now, we need to check that $I_{1}, I_{2}, I_{3}, I_{4}$ tend to 0 independently of $x \in B_{r}$ when $t_{1} \rightarrow t_{2}$.

In fact, by the compactness of the set $g\left(B_{r}\right)$ in view of (2) of Lemma 1, one can deduce that $\lim _{t_{1} \rightarrow t_{2}} I_{1}=0$ uniformly.

Next for $0<h<t_{2}$, when $t_{2}>0$, we similarly derive

$$
\begin{aligned}
& I_{2} \leq \int_{0}^{t_{2}}\left(t_{1}-s\right)^{q-1} s^{n}\left\|\mathscr{S}_{\alpha}\left(t_{1}-s\right)-\mathscr{S}_{\alpha}\left(t_{2}-s\right)\right\|_{\alpha} \\
& \times\|f(s, x(s),(K x)(s),(H x)(s))\|_{\alpha} d s \\
& \leq \int_{0}^{t_{2}-h}\left(t_{2}-s\right)^{q-1} s^{n} \rho(s)\left\|\mathscr{S}_{\alpha}\left(t_{1}-s\right)-\mathscr{S}_{\alpha}\left(t_{2}-s\right)\right\|_{\alpha} d s \\
& +\int_{t_{2}-h}^{t_{2}}\left(t_{2}-s\right)^{q-1} s^{n} \rho(s)\left\|\mathscr{S}_{\alpha}\left(t_{1}-s\right)-\mathscr{S}_{\alpha}\left(t_{2}-s\right)\right\|_{\alpha} d s \\
& \leq \max _{s \in\left[0, t_{2}-h\right]}\left\|\mathscr{S}_{\alpha}\left(t_{1}-s\right)-\mathscr{S}_{\alpha}\left(t_{2}-s\right)\right\|_{\alpha} \int_{0}^{t_{2}-h}\left(t_{2}-s\right)^{q-1} s^{n} \rho(s) d s \\
& +\frac{2 M}{\Gamma(q)} \int_{t_{2}-h}^{t_{2}}\left(t_{2}-s\right)^{q-1} s^{n} \rho(s) d s \\
& \leq \max _{s \in\left[0, t_{2}-h\right]}\left\|\mathscr{S}_{\alpha}\left(t_{1}-s\right)-\mathscr{S}_{\alpha}\left(t_{2}-s\right)\right\|_{\alpha} B\left(\frac{p q-1}{p-1}, \frac{(n+1) p-1}{p-1}\right)^{\frac{p-1}{p}} \\
& \times t_{2}^{\frac{p q+n p-1}{p}}\|\rho\|_{L^{p}\left(J, R^{+}\right)} \\
& +\frac{2 M}{\Gamma(q)} h^{\frac{\chi(p-1)}{(\chi-1) p}} B\left(\frac{\chi(q-1) p}{p-1}+1, \frac{\chi n p}{p-1}+1\right)^{\frac{p-1}{\chi p}} t_{2}^{\frac{\chi p((q-1) p+n)}{p-1}+1}\|\rho\|_{L^{p}\left(J, R^{+}\right)}
\end{aligned}
$$


from which we deduce that $\lim _{\left(h, t_{2}\right) \rightarrow\left(0, t_{1}\right)} I_{2}=0$ uniformly, since by (7) of Lemma 1.

Using the inequality $(x-y)^{a} \leq x^{a}-y^{a}$ for any $x, y \geq 0$ and $a>1$, analogically we derive

$$
\begin{aligned}
& I_{3} \leq \frac{M}{\Gamma(q)} \int_{0}^{t_{2}}\left|\left(t_{1}-s\right)^{q-1}-\left(t_{2}-s\right)^{q-1}\right| s^{n} \\
& \times\|f(s, x(s),(K x)(s),(H x)(s))\|_{\alpha} d s \\
& \leq \frac{M}{\Gamma(q)} \int_{0}^{t_{2}}\left|\left(t_{1}-s\right)^{q-1}-\left(t_{2}-s\right)^{q-1}\right| s^{n} \rho(s) d s \\
& \leq \frac{M}{\Gamma(q)}\left(\int_{0}^{t_{2}} \rho(s)^{p} d s\right)^{\frac{1}{p}}\left(\int_{0}^{t_{2}} s^{\frac{n p}{p-1}}\left|\left(t_{1}-s\right)^{q-1}-\left(t_{2}-s\right)^{q-1}\right|^{\frac{p}{p-1}} d s\right)^{\frac{p-1}{p}} \\
& \leq \frac{M}{\Gamma(q)}\left(\int_{0}^{t_{2}} s^{\frac{n p}{p-1}}\left(\left(t_{2}-s\right)^{\frac{(q-1) p}{p-1}}-\left(t_{1}-s\right)^{\frac{(q-1) p}{p-1}}\right) d s\right)^{\frac{p-1}{p}}\|\rho\|_{L^{p}\left(J, R^{+}\right)} \\
& \leq \frac{M}{\Gamma(q)}\left[B\left(\frac{p q-1}{p-1}, \frac{(n+1) p-1}{p-1}\right)^{\frac{p-1}{p}}\left(t_{2}^{\frac{p q+n p-1}{p}}-t_{1}^{\frac{p q+n p-1}{p}}\right)^{\frac{p-1}{p}}\right. \\
& \left.+\left(\int_{t_{2}}^{t_{1}} s^{\frac{n p}{p-1}}\left(t_{1}-s\right)^{\frac{(q-1) p}{p-1}} d s\right)^{\frac{p-1}{p}}\right]\|\rho\|_{L^{p}\left(J, R^{+}\right)} \\
& \leq \frac{M}{\Gamma(q)}\|\rho\|_{L^{p}\left(J, R^{+}\right)}\left[B\left(\frac{p q-1}{p-1}, \frac{(n+1) p-1}{p-1}\right)^{\frac{p-1}{p}}\right. \\
& \times\left(t_{2}^{\frac{p q+n p-1}{p}}-t_{1}^{\frac{p q+n p-1}{p}}\right)^{\frac{p-1}{p}} \\
& \left.+\left(t_{1}-t_{2}\right)^{\frac{\chi(p-1)}{(\chi-1) p}} B\left(\frac{\chi(q-1) p}{p-1}+1, \frac{\chi n p}{p-1}+1\right)^{\frac{p-1}{\chi p}} t_{1}^{\frac{\chi p((q-1) p+n)}{p-1}+1}\right] \text {. }
\end{aligned}
$$

Thus, $\lim _{t_{1} \rightarrow t_{2}} I_{3}=0$ uniformly.

Finally,

$$
\begin{aligned}
I_{4} & \leq \int_{t_{2}}^{t_{1}}\left(t_{1}-s\right)^{q-1} s^{n}\left\|\mathscr{S}\left(t_{1}-s\right) f(s, x(s),(K x)(s),(H x)(s))\right\|_{\alpha} d s \\
& \leq \frac{M}{\Gamma(q)} \int_{t_{2}}^{t_{1}}\left(t_{1}-s\right)^{q-1} s^{n} \rho(s) d s \\
& \leq \frac{M}{\Gamma(q)}\left(\int_{t_{2}}^{t_{1}} s^{\frac{n p}{p-1}}\left(t_{1}-s\right)^{\frac{(q-1) p}{p-1}} d s\right)^{\frac{p-1}{p}}\|\rho\|_{L^{p}\left(J, R^{+}\right)}
\end{aligned}
$$




$$
\begin{aligned}
\leq & \frac{M}{\Gamma(q)}\left(t_{1}-t_{2}\right)^{\frac{\chi(p-1)}{(\chi-1) p}} B\left(\frac{\chi(q-1) p}{p-1}+1, \frac{\chi n p}{p-1}+1\right)^{\frac{p-1}{\chi p}} \\
& \times t_{1}^{\frac{\chi p((q-1) p+n)}{p-1}+1}\|\rho\|_{L^{p}\left(J, R^{+}\right)}
\end{aligned}
$$

from which we deduce that $\lim _{t_{1} \rightarrow t_{2}} I_{4}=0$ uniformly.

In summary, we have proven that $F\left(B_{r}\right)$ is relatively compact, for $t \in J,\{F x \mid$ $\left.x \in B_{r}\right\}$ is a family of equicontinuous functions. Hence by the Arzela-Aascoli Theorem, $F$ is compact. By Schauder fixed point theorem $F$ has a fixed point $x \in B_{r}$. Consequently, system (1.1) has at least one mild solution on $J$.

Our next result is based on the following well-known fixed point theorem.

Lemma 2 ([18]). Let $\Gamma$ be a condensing operator on a Banach space X. If $\Gamma(\mathscr{B}) \subset \mathscr{B}$ for a convex, closed and bounded set $\mathscr{B}$ of $X$, then $\Gamma$ has a fixed point in $\mathscr{B}$.

Now, we assume the following conditions and apply the above fixed point theorem.

[Hf3]: (1) There exists $\mu$ with $0 \leq \mu \leq \alpha \leq 1$ such that $f: J \times X_{\alpha} \times X_{\alpha} \times X_{\alpha} \rightarrow$ $X_{\mu}$ is continuous and there exist $L_{f}^{(1)}, L_{f}^{(2)}, L_{f}^{(3)}>0$ such that

$$
\begin{aligned}
\left\|f\left(t, x_{1}, x_{2}, x_{3}\right)-f\left(t, y_{1}, y_{2}, y_{3}\right)\right\|_{\mu} \leq L_{f}^{(1)}\left\|x_{1}-y_{1}\right\|_{\alpha} & +L_{f}^{(2)}\left\|x_{2}-y_{2}\right\|_{\alpha} \\
& +L_{f}^{(3)}\left\|x_{3}-y_{3}\right\|_{\alpha}
\end{aligned}
$$

for all $x_{i}, y_{i} \in X_{\alpha}, i=1,2,3$ and $t \in J$.

(2) There exist two positive constants $c^{(1)}, d^{(1)}$ such that for each $(t, x, y, z) \in$ $J \times X_{\alpha} \times X_{\alpha} \times X_{\alpha}$

$$
\|f(t, x, y, z)\|_{\mu} \leq c^{(1)}\left(\|x\|_{\alpha}+\|y\|_{\alpha}+\|z\|_{\alpha}\right)+d^{(1)} .
$$

[Hk3]: (1) The function $k: D_{k} \times X_{\alpha} \rightarrow X_{\alpha}$ is continuous and there exists a $L_{k}^{(1)}>$ 0 such that for $(t, s) \in D_{k}$ and $x, y \in X_{\alpha}$,

$$
\left\|\int_{0}^{t}[k(t, s, x)-k(t, s, y)] d s\right\|_{\alpha} \leq L_{k}^{(1)}\|x-y\|_{\alpha} .
$$

(2) There exists a constant $L_{k}^{(2)}>0$ such that for $(t, s) \in D_{k}$ and $x, y \in X_{\alpha}$,

$$
\left\|\int_{0}^{t} k(t, s, x) d s\right\|_{\alpha} \leq L_{k}^{(2)}\left(1+\|x\|_{\alpha}\right)
$$

[Hh3]: (1) The function $h: D_{h} \times X_{\alpha} \rightarrow X_{\alpha}$ is continuous and there exists a $L_{h}^{(1)}>$ 0 such that for $(t, s) \in D_{h}$ and $x, y \in X_{\alpha}$,

$$
\left\|\int_{0}^{T}[h(t, s, x)-h(t, s, y)] d s\right\|_{\alpha} \leq L_{h}^{(1)}\|x-y\|_{\alpha} .
$$


(2) There exists a constant $L_{h}^{(2)}>0$ such that for $(t, s) \in D_{h}$ and $x, y \in X_{\alpha}$,

$$
\left\|\int_{0}^{T} h(t, s, x) d s\right\|_{\alpha} \leq L_{h}^{(2)}\left(1+\|x\|_{\alpha}\right) .
$$

[Hg3]: $g: C_{\alpha} \rightarrow X_{\alpha}$ is compact continuous and there exists a nondecreasing function $\phi: R^{+} \rightarrow R^{+}$such that for all $x \in C_{\alpha}$,

$$
\|g(x)\|_{\alpha} \leq \phi\left(\|x\|_{\infty}\right) \text {, and } \lim _{l \rightarrow+\infty} \inf \frac{\phi(l)}{l}=\delta<\infty .
$$

Now we are ready to state and prove the following existence result.

Theorem 3. Assume that the conditions [Hf3], [Hk3], [Hh3], [Hg3] are satisfied. If $x_{0} \in X_{\alpha}$ then system (1.1) admits at least one mild solution on $J$ provided that

$$
M\left\{\delta+\frac{\left\|A^{\alpha-\mu}\right\| \Gamma(n)}{\Gamma(n+q+1)}\left[c^{(1)}\left(1+L_{k}^{(2)}+L_{h}^{(2)}\right)\right] T^{n+q}\right\}<1
$$

and

$$
\frac{M\left\|A^{\alpha-\mu}\right\| \Gamma(n)}{\Gamma(n+q+1)} T^{n+q}\left(L_{f}^{(1)}+L_{f}^{(2)} L_{k}^{(1)}+L_{f}^{(3)} L_{h}^{(1)}\right)<1 .
$$

Proof. Define the operator $\Gamma: C_{\alpha} \rightarrow C_{\alpha}$ given by (3.1). For each positive number $l$, let $B_{l}=\left\{x \in C_{\alpha} \mid\|x\|_{\infty} \leq l\right\}$ then, for each $l, B_{l}$ is obviously a bounded closed convex set in $C_{\alpha}$.

First, we claim that $\Gamma\left(B_{l}\right) \subset B_{l}$ for some $l>0$. If it is not true, then for each $l>0$, there would exist $x_{l} \in B_{l}$ and $t_{l} \in J$ such that $\left\|\left(\Gamma x_{l}\right)\left(t_{l}\right)\right\|_{\alpha}>l$. However, on the other hand, by [Hf3], [Hk3] and [Hh3]

$$
\begin{gathered}
l \leq\left\|\left(\Gamma x_{l}\right)\left(t_{l}\right)\right\|_{\alpha} \leq\left\|\mathscr{T}(t)\left(x_{0}+g\left(x_{l}\right)\right)\right\|_{\alpha} \\
+\int_{0}^{t_{l}}\left(t_{l}-s\right)^{q-1} s^{n}\left\|\mathscr{S}\left(t_{l}-s\right) f\left(s, x_{l}(s),\left(K x_{l}\right)(t),\left(H x_{l}\right)(t)\right)\right\|_{\alpha} d s \\
\leq M\left(\left\|x_{0}\right\|_{\alpha}+\left\|g\left(x_{l}\right)\right\|_{\alpha}\right) \\
+\frac{M\left\|A^{\alpha-\mu}\right\|}{\Gamma(q)}\left[c^{(1)}\left(l+L_{k}^{(2)}(1+l)+L_{h}^{(2)}(1+l)+d^{(1)}\right] \int_{0}^{t_{l}}\left(t_{l}-s\right)^{q-1} s^{n} d s\right. \\
\leq M\left(\left\|x_{0}\right\|_{\alpha}+\phi(l)\right) \\
+\frac{\left\|A^{\alpha-\mu}\right\| M}{\Gamma(q)}\left[c^{(1)}\left(l+L_{k}^{(2)}(1+l)+L_{h}^{(2)}(1+l)+d^{(1)}\right] B(q, n+1) t_{l}^{n+q}\right. \\
\quad \leq M\left(\left\|x_{0}\right\|_{\alpha}+\phi(l)\right) \\
+\frac{M\left\|A^{\alpha-\mu}\right\| \Gamma(n)}{\Gamma(n+q+1)}\left[c^{(1)}\left(l+L_{k}^{(2)}(1+l)+L_{h}^{(2)}(1+l)+d^{(1)}\right] T^{n+q} .\right.
\end{gathered}
$$


Dividing both sides by $l$ and taking the lower limit as $l \rightarrow+\infty$, we obtain

$$
1 \leq M\left\{\delta+\frac{\left\|A^{\alpha-\mu}\right\| \Gamma(n)}{\Gamma(n+q+1)}\left[c^{(1)}\left(1+L_{k}^{(2)}+L_{h}^{(2)}\right)\right] T^{n+q}\right\},
$$

which contradicts the expression (3.4). Thus, for some positive number $l, \Gamma\left(B_{l}\right) \subset$ $B_{l}$.

We decompose $\Gamma=\Gamma_{1}+\Gamma_{2}$ as

$$
\begin{aligned}
& \left(\Gamma_{1} x\right)(t)=\mathscr{T}(t)\left[x_{0}+g(x)\right], \\
& \left(\Gamma_{2} x\right)(t)=\int_{0}^{t}(t-s)^{q-1} s^{n} \mathscr{S}(t-s) f(s, x(s),(K x)(s),(H x)(s)) d s .
\end{aligned}
$$

Second, we show that $\Gamma_{1}$ is compact continuous and $\Gamma_{2}$ is a contraction. By [Hg3], we can infer that $\Gamma_{1}$ is compact continuous on $X_{\alpha}$. Next, we prove that $\Gamma_{2}$ is a contraction on $B_{l}$. In fact, for each $t \in J, x, y \in B_{l}$, by [Hg3] we have

$$
\begin{aligned}
& \left\|\left(\Gamma_{2} x\right)(t)-\left(\Gamma_{2} y\right)(t)\right\|_{\alpha} \\
& \leq\left\|A^{\alpha-\mu}\right\| \frac{M}{\Gamma(q)} \int_{0}^{t}(t-s)^{q-1} s^{n} \| f(s, x(s),(K x)(s),(H x)(s)) \\
& \quad-f(s, y(s),(K y)(s),(H y)(s)) \|_{\mu} d s \\
& \leq\left\|A^{\alpha-\mu}\right\| \frac{M}{\Gamma(q)} \int_{0}^{t}(t-s)^{q-1} s^{n}\left[L_{f}^{(1)}\|x(s)-y(s)\|_{\alpha}\right. \\
& \left.\quad+L_{f}^{(2)}\|(K x)(s)-(K y)(s)\|_{\alpha}+L_{f}^{(3)}\|(H x)(s)-(H y)(s)\|_{\alpha}\right] d s \\
& \leq\left\|A^{\alpha-\mu}\right\| \frac{M}{\Gamma(q)}\left(L_{f}^{(1)}+L_{f}^{(2)} L_{k}^{(1)}+L_{f}^{(3)} L_{h}^{(1)}\right) \int_{0}^{t}(t-s)^{q-1} s^{n} d s \|_{x-y \|_{\infty}} \\
& \leq\left\|A^{\alpha-\mu}\right\| \frac{M \Gamma(n)}{\Gamma(n+q+1)} T^{n+q}\left(L_{f}^{(1)}+L_{f}^{(2)} L_{k}^{(1)}+L_{f}^{(3)} L_{h}^{(1)}\right)\|x-y\|_{\infty} .
\end{aligned}
$$

Thus,

$$
\begin{gathered}
\left\|\Gamma_{2} x-\Gamma_{2} y\right\|_{\infty} \leq \frac{M\left\|A^{\alpha-\mu}\right\| \Gamma(n)}{\Gamma(n+q+1)} T^{n+q}\left(L_{f}^{(1)}+L_{f}^{(2)} L_{k}^{(1)}+L_{f}^{(3)} L_{h}^{(1)}\right) \\
\times\|x-y\|_{\infty},
\end{gathered}
$$

which implies that $\Gamma_{2}$ is a contraction by (3.5).

At last, we can conclude that $\Gamma=\Gamma_{1}+\Gamma_{2}$ is a condensing map on $B_{l}$. By Lemma 2, system (1.1) admits at least one mild solution on $J$.

\section{An EXAMPLE}

In this section, we present an example, which indicate how our theorems can be applied to concrete problems. 
Consider the following problem:

$$
\left\{\begin{array}{l}
{ }^{c} D_{t}^{q} x(t, y)-\Delta x(t, y) \\
=\left(\frac{e^{-t}}{e^{t}+e^{-t}}+e^{-t}\right) \cos \left[x(t, y)+\int_{0}^{t} \sin (t+s) x(s, y) d s\right. \\
\quad y \in \Omega, t, s \in(0, T], q=\frac{19}{20}, \\
\left.\quad+\int_{0}^{T} \cos (t s) x(s, y) d s\right], \\
\left.x(t, y)\right|_{y \in \partial \Omega}=0, \quad t>0, \\
x(0, y)=\int_{\Omega} \int_{0}^{T} h(t, y) \log \left(1+|x(t, \xi)|^{\frac{1}{2}}\right) d t d \xi,
\end{array}\right.
$$

where $\Delta$ is the Laplace operator in $\mathbb{R}^{3}, \Omega \subset \mathbb{R}^{3}$ is a bounded domain, $\partial \Omega \in C^{3}$, and $h(t, y) \in C(J \times \bar{\Omega})$.

We apply Theorem 2 by taking $X=L^{2}(\Omega), D(A)=H^{2}(\Omega) \bigcap H_{0}^{1}(\Omega)$, and $A x=-\Delta x$ for $x \in D(A)$ and set $\alpha=0$.

Define $x(t)(y)=x(t, y),(K x)(t)(y)=\int_{0}^{t} \sin (t+s) x(s, y) d s,(H x)(t)(y)=$ $\int_{0}^{T} \cos (t s) x(s, y) d s$, and

$$
\begin{gathered}
f(t, x(t),(K x)(t),(H x)(t))(y) \\
=\left(\frac{e^{-t}}{e^{t}+e^{-t}}+e^{-t}\right) \cos \left[x(t)+\int_{0}^{t} \sin (t+s) x(s) d s+\int_{0}^{T} \cos (t s) x(s) d s\right](y), \\
g(x)(y)=\int_{\Omega} \int_{0}^{T} h(t, y) \log \left(1+|x(t, \xi)|^{\frac{1}{2}}\right) d t d \xi, y \in \bar{\Omega}, x \in C(J, X) .
\end{gathered}
$$

Then, $A$ generates a compact analytic semigroup in $X$ with $M=1$, and

$$
\|f(t, x(t),(K x)(t),(H x)(t))\| \leq \rho(t)=\left(\frac{e^{-t}}{e^{t}+e^{-t}}+e^{-t}\right)(\operatorname{mes}(\Omega))^{\frac{1}{2}}
$$

with $\rho \in L^{p}\left(J, R^{+}\right), p=10$. Moreover, $g$ is compact (see [20]). Next, using $\log (1+$ $a) \leq a$ for any $a \geq 0$, we derive

$$
\int_{\Omega} \log \left(1+|x(\xi)|^{\frac{1}{2}}\right) d \xi \leq \int_{\Omega}|x(\xi)|^{\frac{1}{2}} d \xi \leq(\operatorname{mes}(\Omega))^{\frac{3}{4}}\|x\|^{\frac{1}{2}} \leq \frac{(\operatorname{mes}(\Omega))^{\frac{3}{4}}}{2}(1+\|x\|)
$$

for any $x \in X$. Hence using $\|x(t, \cdot)\| \leq\|x\|_{\infty}$ for any $x \in C(J, X)$, we obtain

$$
\|g(x)\| \leq \frac{T}{2}(\operatorname{mes}(\Omega))^{\frac{7}{4}} \max _{t \in J, y \in \bar{\Omega}}|h(t, y)|\left(1+\|x\|_{\infty}\right), x \in C(J, X) .
$$

Thus problem (4.1) can be rewritten as

$$
\left\{\begin{array}{l}
{ }^{c} D_{t}^{q} x(t)=-A x(t)+t^{n} f(t, x(t),(K x)(t),(H x)(t)), \\
x(0)=g(x)+x_{0} .
\end{array}\right.
$$


Obviously, $q=\frac{19}{20}>\frac{1}{10}=\frac{1}{p}$. Furthermore, if $T$ and $h(t, y)$ satisfy

$$
T(\operatorname{mes}(\Omega))^{\frac{7}{4}} \max _{t \in J, y \in \bar{\Omega}}|h(t, y)|<2
$$

then all the assumptions given in Theorem 2 are verified. Therefore, the problem (4.1) has at least one mild solution.

\section{REFERENCES}

[1] R. P. Agarwal, M. Benchohra, and S. Hamani, "A survey on existence results for boundary value problems of nonlinear fractional differential equations and inclusions," Acta Appl. Math., vol. 109, no. 3, pp. 973-1033, 2010.

[2] B. Ahmad and J. J. Nieto, "Existence of solutions for anti-periodic boundary value problems involving fractional differential equations via Leray-Schauder degree theory," Topol. Methods Nonlinear Anal., vol. 35, no. 2, pp. 295-304, 2010.

[3] Z. Bai, "On positive solutions of a nonlocal fractional boundary value problem," Nonlinear Anal., Theory Methods Appl., Ser. A, Theory Methods, vol. 72, no. 2, pp. A, 916-924, 2010.

[4] K. Balachandran and J. Y. Park, "Nonlocal Cauchy problem for abstract fractional semilinear evolution equations," Nonlinear Anal., Theory Methods Appl., Ser. A, Theory Methods, vol. 71, no. 10, pp. A, 4471-4475, 2009.

[5] M. Benchohra, J. Henderson, S. K. Ntouyas, and A. Ouahab, "Existence results for fractional order functional differential equations with infinite delay," J. Math. Anal. Appl., vol. 338, no. 2, pp. 1340-1350, 2008.

[6] Y.-K. Chang, V. Kavitha, and M. M. Arjunan, "Existence and uniqueness of mild solutions to a semilinear integrodifferential equation of fractional order," Nonlinear Anal., Theory Methods Appl., Ser. A, Theory Methods, vol. 71, no. 11, pp. A, 5551-5559, 2009.

[7] F. Chen, J. J. Neito, and Y. Zhou, "Global attractivity for nonlinear fractional differential equations," Nonlinear Anal.:RWA, vol. 13, no. 1, pp. 287-298, 2012.

[8] K. Diethelm and A. D. Freed, "On the solution of nonlinear fractional order differential equations used in the modeling of viscoelasticity," in Scientific Computing in Chemical Engineering II-Computational Fluid Dynamics, ser. Reaction Engineering and Molecular Properties, F. Keil, W. Mackens, H. Voss, and J. Werther, Eds. Heidelberg: Springer-Verlag, 1999, pp. 217-224.

[9] K. Diethelm, The analysis of fractional differential equations. An application-oriented exposition using differential operators of Caputo type, ser. Lecture Notes in Mathematics. Berlin: Springer, 2010.

[10] M. M. El-Borai, "Semigroups and some nonlinear fractional differential equations," Appl. Math. Comput., vol. 149, no. 3, pp. 823-831, 2004.

[11] L. Gaul, P. Klein, and S. Kempfle, "Damping description involving fractional operators," Mech. Syst. Signal Process., vol. 5, no. 2, pp. 81-88, 1991.

[12] W. G. Glockle and T. F. Nonnenmacher, "A fractional calculus approach of self-similar protein dynamics," Biophys. J., vol. 68, no. 1, pp. 46-53, 1995.

[13] J. Henderson and A. Ouahab, "Fractional functional differential inclusions with finite delay," Nonlinear Anal., Theory Methods Appl., vol. 70, no. 5, pp. A, 2091-2105, 2009.

[14] R. Hilfer, Ed., Applications of fractional calculus in physics. Singapore: World Scientific, 2000.

[15] L. Hu, Y. Ren, and R. Sakthivel, "Existence and uniqueness of mild solutions for semilinear integro-differential equations of fractional order with nonlocal initial conditions and delays," Semigroup Forum, vol. 79, no. 3, pp. 507-514, 2009. 
[16] O. K. Jaradat, A. Al-Omari, and S. Momani, "Existence of the mild solution for fractional semilinear initial value problems," Nonlinear Anal., Theory Methods Appl., vol. 69, no. 9, pp. A, 3153-3159, 2008.

[17] A. A. Kilbas, H. M. Srivastava, and J. J. Trujillo, Theory and applications of fractional differential equations, ser. North-Holland Mathematics Studies. Amsterdam: Elsevier, 2006, vol. 204.

[18] V. Lakshmikantham and S. Leela, Differential and integral inequalities. Theory and applications. Vol. I: Ordinary differential equations, ser. Mathematics in Science and Engineering. New YorkLondon: Academic Press, 1969, vol. 55.

[19] V. Lakshmikantham, S. Leela, and J. Vasundhara Devi, Theory of fractional dynamic systems. Cambridge: Cambridge Scientific Publishers, 2009.

[20] J. Liang, J. H. Liu, and T.-J. Xiao, "Nonlocal problems for integrodifferential equations," Dyn. Contin. Discrete Impuls. Syst., Ser. A, Math. Anal., vol. 15, no. 6, pp. 815-824, 2008.

[21] H. Liu and J.-C. Chang, "Existence for a class of partial differential equations with nonlocal conditions," Nonlinear Anal., Theory Methods Appl., vol. 70, no. 9, pp. A, 3076-3083, 2009.

[22] F. Mainardi, "Fractional calculus, some basic problems in continuum and statistical mechanics," in Fractals and Fractional Calculus in Continuum Mechanics, A. Carpinteri and F. Mainardi, Eds. Wien: Springer-Verlag, 1997, pp. 291-348.

[23] F. Metzler, W. Schick, H. G. Kilian, and T. F. Nonnenmache, "Relaxation in filled polymers: A fractional calculus approach," J. Chem. Phys., vol. 103, no. 16, pp. 7180-7186, 1995.

[24] M. W. Michalski, "Derivatives of noninteger order and their applications," Diss. Math., vol. 328, p. 47, 1993.

[25] K. S. Miller and B. Ross, An introduction to the fractional calculus and fractional differential equations. New York: John Wiley \& Sons, Inc., 1993.

[26] G. M. Mophou and G. M. N'Guérékata, "Existence of the mild solution for some fractional differential equations with nonlocal conditions," Semigroup Forum, vol. 79, no. 2, pp. 315-322, 2009.

[27] G. M. N'Guérékata, "A cauchy problem for some fractional abstract differential equation with non local conditions," Nonlinear Anal., Theory Methods Appl., vol. 70, no. 5, pp. A, 1873-1876, 2009.

[28] A. Pazy, Semigroups of linear operators and applications to partial differential equations, ser. Applied Mathematical Sciences. New York: Springer-Verlag, 1983, vol. 44.

[29] I. Podlubny, Fractional differential equations. An introduction to fractional derivatives, fractional differential equations, to methods of their solution and some of their applications, ser. Mathematics in Science and Engineering. San Diego, CA: Academic Press, 1999, vol. 198.

[30] V. E. Tarasov, Fractional dynamics. Applications of fractional calculus to dynamics of particles, fields and media, ser. Nonlinear Physical Science. Berlin-Beijing: Springer-Higher Education Press, 2010.

[31] J. Wang, X. Xiang, and W. Wei, "A class of nonlinear integrodifferential impulsive periodic systems of mixed type and optimal controls on Banach space," J. Appl. Math. Comput., vol. 34, no. 1-2, pp. 465-484, 2010.

[32] J. Wang and Y. Zhou, "Analysis of nonlinear fractional control systems in Banach spaces," Nonlinear Anal., Theory Methods Appl., Ser. A, Theory Methods, vol. 74, no. 17, pp. 5929-5942, 2011.

[33] W. Wei, X. Xiang, and Y. Peng, "Nonlinear impulsive integro-differential equations of mixed type and optimal controls," Optimization, vol. 55, no. 1-2, pp. 141-156, 2006.

[34] S. Zhang, "Existence of positive solution for some class of nonlinear fractional differential equations," J. Math. Anal. Appl., vol. 278, no. 1, pp. 136-148, 2003.

[35] Y. Zhou and F. Jiao, "Existence of mild solutions for fractional neutral evolution equations," Comput. Math. Appl., vol. 59, no. 3, pp. 1063-1077, 2010. 
Authors' addresses

\section{JinRong Wang}

Department of Mathematics, Guizhou University, Guiyang, Guizhou 550025, P.R. China

E-mail address: wjr9668@126.com

\section{Wei Wei}

Department of Mathematics, Guizhou University, Guiyang, Guizhou 550025, P.R. China

E-mail address: wwei@gzu.edu.cn

\section{Michal Fečkan}

Department of Mathematical Analysis and Numerical Mathematics, Faculty of Mathematics, Physics and Informatics, Comenius University, Mlynská dolina, 84248 Bratislava, Slovakia and Mathematical Institute, Slovak Academy of Sciences, Štefánikova 49, 81473 Bratislava, Slovakia

E-mail address: Michal.Feckanefmph.uniba.sk 